\title{
Cyclization Reactions of Hydrazones XXVII" Synthesis and oxidative cyclization of some formazan-substituted quinoxaline-2-ones
}

\author{
Iveta Wiedermannová. Jan Slouka \\ Department of Organic Chemistry. Palacky University, 77146 Olomouc, Czech Republic \\ E-mail: Wiedermannova (dpprfnw.upol.cz
}

\begin{abstract}
A series of 1,5-diaryl-3-formazylglyoxylic acids 1a-1f was prepared by azocoupling of diazonium salts with sodium pyruvate. These compounds gave the corresponding 3-(1,5-diaryl-3-formazvl)-1,2-dihydro-quinoxaline-2-ones $\mathbf{2 a - 2 f}$ by condensation with o-phenylenediamine. Oxidative cyclization of these compounds did not lead to the $[1,2,3]$ triazolo[1,5-a]quinoxaline derivatives $6 \mathbf{a - 6 f}$, but to the isomeric 2,3-diaryl-5-(2-oxo1,2-dihydro-quinoxaline-3-yl)-tetrazolium chlorides $\mathbf{3 a - 3 f}$, which were transformed into the less hygroscopic picrates $\mathbf{4 a - 4 f}$.
\end{abstract}

\section{Introduction}

The hydrazones of some heterocyclic aldehydes and ketones can be transformed to the condensed heterocyclic compounds by oxidative cyclization.

For example, phenylhydrazone of 2-benzoyl-benzothiazole gives 1,3-diphenyl[1,2,3]triazolo[5, l-b]benzothiazolium salts in this way (1) and the same reaction of phenylhydrazone of 2-benzoylbenzimidazole leads to the 1,3-diphenyl-[1,2,3]triazolo[1,5-a]benzimidazole (1). The oxidative cyclization of 2-acylpyridine hydrazones to $[1,2,3]$ triazolo $[1,5-a] p y r i d i n e$ derivatives is also known (2).

In this connection we were interested in properties of 3-(1,5-diaryl-3-formazyl)-1,2dihydro-quinoxaline-2-one, for which it was possible to expect two different courses of oxidative cyclization: usual cyclization to the corresponding tetrazolium salts 3 , or oxidative cyclization, which could lead to the isomeric derivatives of $[1,2,3]$ triazolo[1,5-a]quinoxalinium salts $\mathbf{6}$. The isomerization of the Z-hydrazono form $\mathbf{2}$ into the E-isomer $\mathbf{5}$ is possible due to azo-hydrazono tautomerism.

\section{Results}

Contrary to the series of heterocyclic methyl-derivatives, which gave corresponding formazans by azocoupling with two equivalents of diazonium salts (3), in the case of 3-methyl-1,2-dihydro-quinoxaline-2-one this reaction didn't occur and gave arylhydrazones of 2-oxo-1,2-dihydro-quinoxaline-3-carbaldehyde only (4-9). These arylhydrazones are not able to react with another equivalent of diazonium salts to form formazans (9).

For this reason we had to prepare these 3-(1,5-diaryl-3-formazyl)-1,2-dihydroquinoxaline-2-ones in a different way. Using modified Bamberger and Müller method (10) we prepared a series of 1,5-diaryl-3-formazylglyoxylic acids 1a-1f by azocoupling of diazonium salts with sodium pyruvate in alkaline medium. These compounds were condensed with o-phenylenediamine and we received a series of formazanes $2 \mathbf{a}-2 \mathbf{f}$ mentioned above.

For oxidative cyclization of formazanes we used lead(IV)tetraacetate in a chloroform solution. With respect to the fact that products are nearly colourless crystalline compounds and there is no characteristic band for arylazo group in the visible part of UV-VIS spectrum, it is possible to exclude the structure expressed by formula 6 , so that studied compounds are

\footnotetext{
'Part XXVII: see J. Styskala, J. Slouka: Heter. Commun. $\underline{5}, 349$ (1999)
} 
2.3-diaryl-5-(2-oxo-1,2-dihydro-quinoxaline-3-yl)-tetrazolium chlorides 3a-3f. These compounds are soluble in water, but give after recrystallisation the corresponding hydrates. By the reaction with sodium picrate, the chlorides were transformed to the corresponding picrates 4a-4f, which aren't hygroscopic. By the reduction with sodium hyposulfite. tetrazolium salts $\mathbf{3}$ were transformed back to the corresponding formazans $\mathbf{2}$.

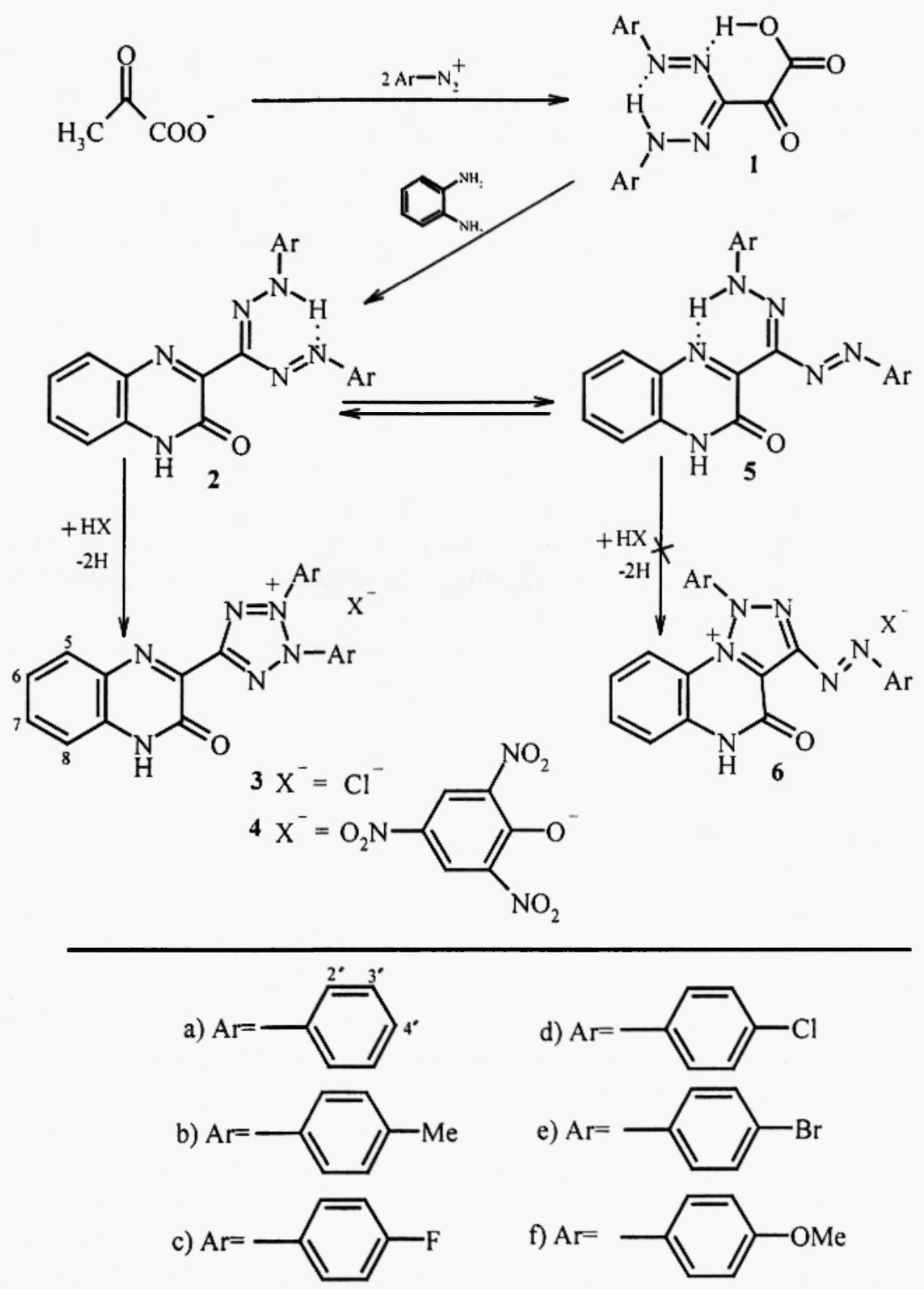




\section{Apparatus and methods}

The melting points were determined on a Boetius apparatus and are uncorrected. The IR spectra were measured using $\mathrm{KBr}$ disc technique and scanned on an ATI Unicam Genesis FTIR instrument. Elemental analyses were performed by using an EA 1108 Elemental Analyser (Fison Instrument). NMR spectra were measured on a Bruker AMX-360 spectrometer $\left(360 \mathrm{MHz}\right.$ ) in DMSO- $\mathrm{d}_{6}$; the chemical shifts $\delta$ are reported in ppm and coupling constants are in Hertz. Electronic spectra were measured in ethanol solutions on a UV-VIS spectrometr Unicam Helios $\alpha$ in $1 \mathrm{~cm}$ cuvettes. Concentrations of the samples varied from $0.5-1.10^{-5} \mathrm{~mol} . \mathrm{l}^{-1}$.

\section{Experimental}

\section{1,5-Diaryl-3-formazylglyoxylic acids (1a-1f)}

General procedure:

A solution of $\mathrm{NaNO}_{2}(1.38 \mathrm{~g}, 20.0 \mathrm{mmol})$ in ice-cold water $(5 \mathrm{ml})$ was added portionwise under stirring to the solution of corresponding aromatic amine $(20.2 \mathrm{mmol})$ in a mixture of $\mathrm{HCl}(37 \%, 10.0 \mathrm{ml})$ and water $(10-20 \mathrm{ml})$, which was cooled in an ice-bath. The solution was left to stand for $20 \mathrm{~min}$ in an ice bath and then was added portionwise during $3 \mathrm{~min}$. under stirring to a pre-cooled solution of sodium pyruvate $(4.00 \mathrm{~g}, 36,35 \mathrm{mmol})$ and $\mathrm{KOH}(30 \mathrm{~g})$ in water $(300 \mathrm{ml})$. Reaction mixture was left in an ice bath for $20 \mathrm{~min}$. with vigourous stirring. Small amount of separated precipitate of 1,5-diaryl-3-arylazoformazane was filtered off and washed with water. The combined filtrates were acidified under vigorous stirring with $\mathrm{HCl}(37 \%)$ to $\mathrm{pH}=1$. After several hours, the separated red compound was collected with suction, thoroughly washed with water and suspended in a solution of $\mathrm{NaHCO}_{3}$ $(1.0 \mathrm{~g})$ in water $(30-50 \mathrm{ml})$. The reaction mixture was stirred for several hours and then filtered after addition of small amount of charcoal. The filtrate was acidified with $\mathrm{HCl}(37 \%)$ to $\mathrm{pH}=1$. Precipitate was collected with suction after several hours, carefully washed with water and dried in air. Obtained compounds were purified by recrystallization from ethanol/water.

For further details see tables 1 and 2.

\section{3-(1,5-Diaryl-3-formazyl)-1,2-dihydro-quinoxaline-2-ones (2a-2f)}

General procedure:

Formazylglyoxylic acid 1a-1f $(1.0 \mathrm{mmol})$ was disolved in hot ethanol $(10 \mathrm{ml})$ and o-phenylenediamine $(108 \mathrm{mg}, 1.0 \mathrm{mmol})$ was disolved in $1 \mathrm{ml}$ hot ethanol. The mixture of both solutions was refluxed for $15 \mathrm{~min}$. After cooling to $20^{\circ} \mathrm{C}$, the red crystalline compound was separated with suction, washed with water and dried. Obtained compounds were purified by recrystallization from ethanol.

For further details see table 1 and 2.

\section{2,3-Diaryl-5-(2-oxo-1,2-dihydro-quinoxaline-3-yl)-tetrazolium chlorides (3a-3f)}

General procedure:

Lead(IV)tetraacetate $(0.50 \mathrm{~g}, 1.12 \mathrm{mmol})$ was added with stirring to a solution prepared from 1,2-dihydro-3-(1,5-diarylformazyl-3-yl-quinoxaline-2-one 2a-2f (1.00 mmol) and $\mathrm{CHCI}_{3} 50-150 \mathrm{ml}$. The solution was filtered after 3 hours of stirring. The filtrate was evaporated, the residue was dissolved in $\mathrm{H}_{2} \mathrm{O}(10 \mathrm{ml})$ and the solution was acidified with $\mathrm{HCl}(37 \%)$ to $\mathrm{pH}=2$. The precipitate was separated with suction and the filtrate was evaporated. The residue was dissolved in methanol $(7-10 \mathrm{ml})$, filtered and evaporated. The 
residue was dried over $\mathrm{KOH}$. Compounds 3c, 3e and $3 f$ were used without purifying and for preparation of corresponding picrates.

For further details see tables 1 and 2.

2.3-Diaryl-5-(2-oxo-1.2-dihydro-quinoxaline-3-yl)-tetrazolium picrates (4a-4f)

General procedure:

A solution of sodium picrate $(251.0 \mathrm{mg} .1 .00 \mathrm{mmol})$ in $\mathrm{H}_{2} \mathrm{O}(5 \mathrm{ml})$ was added to the stirred solution prepared from tetrazolium salt 3a-3f $(1 \mathrm{mmol})$ and $\mathrm{H}_{2} \mathrm{O}(1-3 \mathrm{ml})$. The precipitated compound $\mathbf{4 a - 4 f}$ was collected with suction and dried.

For further details see tables 1 and 2.

Table 1

${ }^{\mathrm{I}} \mathrm{H}-\mathrm{NMR}$ spectra of compounds 1,2 and $3 \mathrm{a}$

\begin{tabular}{|c|l|}
\hline Compound & \multicolumn{1}{|c|}{${ }^{\mathrm{H}-\mathrm{NMR} \text { spectrum }}$} \\
\hline 1a & $7.50\left(\mathrm{t}, 2 \mathrm{H}, \mathrm{J}=7.5, \mathrm{H}_{4}\right) ; 7.61\left(\mathrm{t}, 4 \mathrm{H}, \mathrm{J}=8.0, \mathrm{H}_{3}\right) ; 7.88\left(\mathrm{~d}, 4 \mathrm{H}, \mathrm{J}=7.5, \mathrm{H}_{2}\right) ; 14.48(\mathrm{~s}, 1 \mathrm{H}, \mathrm{NH})$ \\
\hline 1b & $2.43\left(\mathrm{~s}, 6 \mathrm{H}, \mathrm{CH}_{3}\right) ; 7.41\left(\mathrm{~d}, 4 \mathrm{H}, \mathrm{J}=8.4, \mathrm{H}_{3}\right) ; 7.78\left(\mathrm{~d}, 4 \mathrm{H}, \mathrm{J}=8.4, \mathrm{H}_{2}\right) ; 14.7(\mathrm{~s}, 1 \mathrm{H}, \mathrm{NH})$ \\
\hline 1c & $7.47\left(\mathrm{t} .4 \mathrm{H}, \mathrm{J}=8.8 . \mathrm{H}_{3}\right) ; 7.94(\mathrm{dd}, 4 \mathrm{H}, \mathrm{J}=3.4, \mathrm{~J}=5.1, \mathrm{H}) ; 14.32(\mathrm{~s}, 1 \mathrm{H}, \mathrm{NH})$ \\
\hline 1d & $7.68\left(\mathrm{~d} .4 \mathrm{H} . \mathrm{J}=8.8 . \mathrm{H}_{3}\right) ; 7.90\left(\mathrm{~d} .4 \mathrm{H} . \mathrm{J}=8.8, \mathrm{H}_{2}\right) ; 14.21(\mathrm{~s}, 1 \mathrm{H}, \mathrm{NH})$ \\
\hline 1e & $7.69\left(\mathrm{~d}, 4 \mathrm{H}, \mathrm{J}=8.7, \mathrm{H}_{3}\right) ; 7.91\left(\mathrm{~d}, 4 \mathrm{H}, \mathrm{J}=8.7, \mathrm{H}_{2}\right) ; 14.20(\mathrm{~s}, 1 \mathrm{H}, \mathrm{NH})$ \\
\hline 1f & $3.89\left(\mathrm{~s}, 6 \mathrm{H}, \mathrm{CH}_{3}\right) ; 7.16\left(\mathrm{dd}, 4 \mathrm{H}, \mathrm{J}=7.2, \mathrm{~J}=2.1, \mathrm{H}_{3}\right) ; 7.84\left(\mathrm{dd}, 4 \mathrm{H}, \mathrm{J}=7.2, \mathrm{~J}=2.1, \mathrm{H}_{2}\right) ; 14.80(\mathrm{~s}, 1 \mathrm{H}, \mathrm{NH})$ \\
\hline 2a & $7.40(\mathrm{t}, 4 \mathrm{H}, \mathrm{J}=7.3, \mathrm{ArH}) ; 7.61(\mathrm{t}, 4 \mathrm{H}, \mathrm{J}=7.3, \mathrm{ArH}) ; 7.92(\mathrm{~d}, 6 \mathrm{H}, \mathrm{J}=6.7, \mathrm{ArH}), 13.97(\mathrm{~s}, 2 \mathrm{H}, \mathrm{NH})$ \\
\hline 2b & $2.43\left(\mathrm{~s}, 6 \mathrm{H}, \mathrm{CH}_{3}\right) ; 7.40(\mathrm{~m}, 6 \mathrm{H}, \mathrm{ArH}) ; 7.87(\mathrm{~m}, 6 \mathrm{H}, \mathrm{ArH}) ; 13.92(\mathrm{Brs}, 2 \mathrm{H}, \mathrm{NH})$ \\
\hline 2c & $7.43(\mathrm{~m}, 6 \mathrm{H}, \mathrm{ArH}) ; 7.96(\mathrm{~m}, 6 \mathrm{H}, \mathrm{ArH}) ; 13.96(\mathrm{Brs}, 2 \mathrm{H}, \mathrm{NH})$ \\
\hline 2d & $7.60(\mathrm{~m}, 6 \mathrm{H}, \mathrm{ArH}) ; 7.88(\mathrm{~m}, 6 \mathrm{H}, \mathrm{ArH}) ; 14.06(\mathrm{Brs}, 2 \mathrm{H} \mathrm{NH})$ \\
\hline 2e & $7.65(\mathrm{~m}, 6 \mathrm{H}, \mathrm{ArH}) ; 7.93(\mathrm{~m}, 6 \mathrm{H}, \mathrm{ArH}) ; 13.94(\mathrm{~s}, 2 \mathrm{H}, \mathrm{NH})$ \\
\hline 2f & $3.93\left(\mathrm{~s}, 6 \mathrm{H}, \mathrm{CH}_{3}\right) ; 7.41(\mathrm{~m}, 6 \mathrm{H}, \mathrm{ArH}) ; 7.90(\mathrm{~m}, 6 \mathrm{H}, \mathrm{ArH}) ; 13.87(\mathrm{Brs}, 2 \mathrm{H}, \mathrm{NH})$ \\
\hline 3a & $7.48\left(\mathrm{~m}, 2 \mathrm{H}, \mathrm{H}_{4}\right) ; 7.78\left(\mathrm{t}, 4 \mathrm{H}, \mathrm{J}=7.7, \mathrm{H}_{3}\right) ; 7.86\left(\mathrm{~m}, 4 \mathrm{H}, \mathrm{H}_{5.8}\right) ; 8.05\left(\mathrm{~d}, 4 \mathrm{H}, \mathrm{J}=7.7, \mathrm{H}_{2}\right)$ \\
\hline
\end{tabular}


Table 2

Characteristic data of compounds $1-4$

\begin{tabular}{|c|c|c|c|c|c|c|c|c|}
\hline \multirow[t]{2}{*}{ Compound } & \multirow{2}{*}{$\begin{array}{l}\text { M.p. }\left({ }^{\circ} \mathrm{C}\right) \\
\text { Yield (\%) }\end{array}$} & \multirow{2}{*}{$\begin{array}{l}\text { Formula } \\
\text { M.w. }\end{array}$} & \multicolumn{3}{|c|}{$\begin{array}{l}\text { ElementalAnalysis } \\
\text { (Calcul./Found) }\end{array}$} & \multirow[t]{2}{*}{$v(C=0)$} & \multirow[t]{2}{*}{$\lambda(\max )$} & \multirow[t]{2}{*}{$(\log \varepsilon)$} \\
\hline & & & $\% \mathrm{C}$ & $\% \mathrm{H}$ & $\% \mathrm{~N}$ & & & \\
\hline $1 \mathbf{a}$ & \begin{tabular}{|c|}
$165-167^{*}$ \\
68.52 \\
\end{tabular} & $\begin{array}{c}\mathrm{C}_{15} \mathrm{H}_{12} \mathrm{~N}_{4} \mathrm{O}_{3} \\
296.28\end{array}$ & $\begin{array}{l}60.80 \\
60.79 \\
\end{array}$ & $\begin{array}{l}4.08 \\
4.01\end{array}$ & $\begin{array}{l}18.91 \\
17.99\end{array}$ & 1740 & $\begin{array}{l}304 \mathrm{~nm} \\
439 \mathrm{~nm}\end{array}$ & $\begin{array}{l}4.66 \\
4.78\end{array}$ \\
\hline $1 b$ & $\begin{array}{c}173-174 \\
57.00 \\
\end{array}$ & $\begin{array}{c}\mathrm{C}_{17} \mathrm{H}_{16} \mathrm{~N}_{4} \mathrm{O}_{3}, 0.5 \mathrm{H}_{2} \mathrm{O} \\
333.34\end{array}$ & $\begin{array}{l}61.25 \\
61.08 \\
\end{array}$ & $\begin{array}{l}5.14 \\
4.89\end{array}$ & $\begin{array}{l}16,81 \\
16.00\end{array}$ & 1716 & $\begin{array}{l}317 \mathrm{~nm} \\
448 \mathrm{~nm}\end{array}$ & $\begin{array}{l}4.29 \\
4.44\end{array}$ \\
\hline 1c & \begin{tabular}{|c|}
$178-179$ \\
55.08 \\
\end{tabular} & $\begin{array}{c}\mathrm{C}_{15} \mathrm{H}_{10} \mathrm{~F}_{2} \mathrm{~N}_{4} \mathrm{O}_{3} \cdot \mathrm{H}_{2} \mathrm{O} \\
350.29\end{array}$ & $\begin{array}{l}51.43 \\
51.45 \\
\end{array}$ & $\begin{array}{l}3.45 \\
3.35\end{array}$ & $\begin{array}{l}16.00 \\
15.97\end{array}$ & 1717 & $\begin{array}{l}308 \mathrm{~nm} \\
436 \mathrm{~nm}\end{array}$ & $\begin{array}{l}4.44 \\
4.61\end{array}$ \\
\hline $1 d$ & $\begin{array}{c}174-175 \\
65.44 \\
\end{array}$ & $\begin{array}{c}\mathrm{C}_{15} \mathrm{H}_{10} \mathrm{Cl}_{2} \mathrm{~N}_{4} \mathrm{O}_{3} \\
365.19\end{array}$ & $\begin{array}{l}49.33 \\
49.48 \\
\end{array}$ & $\begin{array}{l}2.79 \\
2.95\end{array}$ & $\begin{array}{l}15.34 \\
15.19 \\
\end{array}$ & 1713 & $\begin{array}{l}314 \mathrm{~nm} \\
444 \mathrm{~nm}\end{array}$ & $\begin{array}{l}4.43 \\
4.60\end{array}$ \\
\hline $1 \mathrm{e}$ & \begin{tabular}{|c|}
$185-187$ \\
70.46 \\
\end{tabular} & $\begin{array}{c}\mathrm{C}_{15} \mathrm{H}_{10} \mathrm{Br}_{2} \mathrm{~N}_{4} \mathrm{O}_{3} \\
454.10\end{array}$ & $\begin{array}{l}39.67 \\
39.47 \\
\end{array}$ & $\begin{array}{l}2.22 \\
2.34\end{array}$ & $\begin{array}{l}12.34 \\
11.85\end{array}$ & 1672 & $\begin{array}{l}313 \mathrm{~nm} \\
447 \mathrm{~nm}\end{array}$ & $\begin{array}{l}4.32 \\
4.45\end{array}$ \\
\hline If & $\begin{array}{c}164-165 \\
63.14 \\
\end{array}$ & $\begin{array}{c}\mathrm{C}_{17} \mathrm{H}_{16} \mathrm{~N}_{4} \mathrm{O}_{5} \\
356.33\end{array}$ & $\begin{array}{l}57.30 \\
56.96 \\
\end{array}$ & $\begin{array}{l}4.53 \\
4.76 \\
\end{array}$ & $\begin{array}{l}15.72 \\
15.70 \\
\end{array}$ & 1716 & $\begin{array}{l}310 \mathrm{~nm} \\
440 \mathrm{~nm}\end{array}$ & $\begin{array}{l}4.53 \\
4.68 \\
\end{array}$ \\
\hline $2 a$ & $\begin{array}{c}218-219 \\
91.3 \\
\end{array}$ & $\begin{array}{c}\mathrm{C}_{21} \mathrm{H}_{16} \mathrm{~N}_{6} \mathrm{O} \\
368.4\end{array}$ & $\begin{array}{l}68.47 \\
68.55\end{array}$ & $\begin{array}{l}4.38 \\
4.58\end{array}$ & $\begin{array}{l}22.81 \\
20.92\end{array}$ & 1765 & $\begin{array}{l}322 \mathrm{~nm} \\
438 \mathrm{~nm}\end{array}$ & $\begin{array}{l}5.10 \\
5.04\end{array}$ \\
\hline $2 b$ & $\begin{array}{c}222.224 \\
95.4\end{array}$ & $\begin{array}{c}\mathrm{C}_{3,3} \mathrm{H}_{20} \mathrm{~N}_{6} \mathrm{O} \\
396.45\end{array}$ & $\begin{array}{l}69.68 \\
68.99 \\
\end{array}$ & $\begin{array}{l}5.08 \\
4.99 \\
\end{array}$ & $\begin{array}{l}21.20 \\
20.90\end{array}$ & 1700 & $\begin{array}{l}327 \mathrm{~nm} \\
459 \mathrm{~nm}\end{array}$ & $\begin{array}{l}4.41 \\
4.32\end{array}$ \\
\hline $2 c$ & $\begin{array}{c}221-222 \\
93.5\end{array}$ & $\begin{array}{c}\mathrm{C}_{21} \mathrm{H}_{14} \mathrm{~F}_{2} \mathrm{~N}_{6} \mathrm{O} \\
404.38\end{array}$ & $\begin{array}{l}62.37 \\
62.05\end{array}$ & $\begin{array}{l}3.49 \\
3.20\end{array}$ & $\begin{array}{l}20.78 \\
20.95\end{array}$ & 1768 & $\begin{array}{l}325 \mathrm{~nm} \\
435 \mathrm{~nm}\end{array}$ & $\begin{array}{l}4.40 \\
4.31\end{array}$ \\
\hline $2 d$ & $\begin{array}{c}223-225 \\
97.0\end{array}$ & $\begin{array}{c}\mathrm{C}_{21} \mathrm{H}_{14} \mathrm{Cl}_{2} \mathrm{~N}_{6} \mathrm{O} \\
437.38\end{array}$ & $\begin{array}{l}57.67 \\
57.45\end{array}$ & $\begin{array}{l}3.23 \\
3.23\end{array}$ & $\begin{array}{l}19.21 \\
20.50\end{array}$ & 1768 & $\begin{array}{l}324 \mathrm{~nm} \\
441 \mathrm{~nm}\end{array}$ & $\begin{array}{l}4.42 \\
4.36\end{array}$ \\
\hline $2 e$ & $\begin{array}{c}243-244 \\
90.9\end{array}$ & $\begin{array}{c}\mathrm{C}_{21} \mathrm{H}_{14} \mathrm{Br}_{2} \mathrm{~N}_{6} \mathrm{O} \\
526.18\end{array}$ & $\begin{array}{l}47.93 \\
47.55 \\
\end{array}$ & $\begin{array}{l}2.68 \\
2.45\end{array}$ & $\begin{array}{l}15.97 \\
16.95\end{array}$ & 1742 & $\begin{array}{l}330 \mathrm{~nm} \\
450 \mathrm{~nm}\end{array}$ & $\begin{array}{l}4.11 \\
4.24\end{array}$ \\
\hline $2 f$ & $\begin{array}{c}218-219 \\
94.2\end{array}$ & $\begin{array}{c}\mathrm{C}_{23} \mathrm{H}_{20} \mathrm{~N}_{6} \mathrm{O}_{3} \\
428.45\end{array}$ & $\begin{array}{l}64.48 \\
64.20\end{array}$ & $\begin{array}{l}4.70 \\
4.28 \\
\end{array}$ & $\begin{array}{l}19.61 \\
19.90 \\
\end{array}$ & 1786 & $\begin{array}{l}335 \mathrm{~nm} \\
465 \mathrm{~nm}\end{array}$ & $\begin{array}{l}4.43 \\
4.23 \\
\end{array}$ \\
\hline $3 a$ & $\begin{array}{c}245-255 \\
69.3\end{array}$ & $\begin{array}{c}\mathrm{C}_{21} \mathrm{H}_{15} \mathrm{ClN}_{6} \mathrm{O} .3 .5 \mathrm{H}_{2} \mathrm{O} \\
465.94\end{array}$ & $\begin{array}{l}54.13 \\
54.70\end{array}$ & $\begin{array}{l}4.76 \\
4.25\end{array}$ & $\begin{array}{l}18.04 \\
18.14\end{array}$ & 1728 & $306 \mathrm{~nm}$ & 4.64 \\
\hline $3 \mathbf{b}$ & $\begin{array}{c}210-212 \\
57.2 \\
\end{array}$ & $\begin{array}{c}\mathrm{C}_{33} \mathrm{H}_{19} \mathrm{ClN}_{6} \mathrm{O} \cdot 3 \mathrm{H}_{2} \mathrm{O} \\
484.99 \\
\end{array}$ & $\begin{array}{l}56.96 \\
56.88 \\
\end{array}$ & $\begin{array}{l}5.20 \\
4.56\end{array}$ & $\begin{array}{l}17.33 \\
16.33\end{array}$ & 1710 & $305 \mathrm{~nm}$ & 4.54 \\
\hline $3 d$ & $\begin{array}{c}230-235 \\
70.7\end{array}$ & $\begin{array}{c}\mathrm{C}_{2 !} \mathrm{H}_{13} \mathrm{Cl}_{3} \mathrm{~N}_{6} \mathrm{O} .3 .5 \mathrm{H}_{2} \mathrm{O} \\
534.87\end{array}$ & $\begin{array}{l}47.15 \\
46.89\end{array}$ & $\begin{array}{l}3.77 \\
3.19\end{array}$ & $\begin{array}{l}15.71 \\
14.50\end{array}$ & 1749 & $308 \mathrm{~nm}$ & 4.52 \\
\hline $4 a$ & $\begin{array}{c}127-128 \\
97.9 \\
\end{array}$ & $\begin{array}{c}\mathrm{C}_{27} \mathrm{H}_{17} \mathrm{~N}_{9} \mathrm{O}_{8} \cdot 0.5 \mathrm{H}_{2} \mathrm{O} \\
604.49\end{array}$ & $\begin{array}{l}53.65 \\
53.78 \\
\end{array}$ & $\begin{array}{l}3.00 \\
3.01\end{array}$ & $\begin{array}{l}20.85 \\
20.02\end{array}$ & 1631 & $307 \mathrm{~nm}$ & 4.62 \\
\hline $4 b$ & $\begin{array}{c}129-131 \\
93.04 \\
\end{array}$ & $\begin{array}{c}\mathrm{C}_{29} \mathrm{H}_{21} \mathrm{~N}_{9} \mathrm{O}_{8} \\
623.54\end{array}$ & $\begin{array}{l}55.86 \\
55.69 \\
\end{array}$ & $\begin{array}{l}3.39 \\
3.18 \\
\end{array}$ & $\begin{array}{l}20.22 \\
19.11 \\
\end{array}$ & 1634 & $313 \mathrm{~nm}$ & 4.70 \\
\hline $4 c$ & $\begin{array}{c}119-121 \\
96.0 \\
\end{array}$ & $\begin{array}{c}\mathrm{C}_{27} \mathrm{H}_{15} \mathrm{~F}_{2} \mathrm{~N}_{9} \mathrm{O}_{8} \\
631.47 \\
\end{array}$ & $\begin{array}{l}51.36 \\
51.45 \\
\end{array}$ & $\begin{array}{l}2.39 \\
2.22 \\
\end{array}$ & $\begin{array}{l}19.96 \\
18.82 \\
\end{array}$ & 1634 & $309 \mathrm{~nm}$ & 4.70 \\
\hline $4 d$ & $\begin{array}{c}134-135 \\
90.3\end{array}$ & $\begin{array}{c}\mathrm{C}_{27} \mathrm{H}_{15} \mathrm{Cl}_{2} \mathrm{~N}_{9} \mathrm{O}_{8} \\
664.47\end{array}$ & $\begin{array}{l}48.81 \\
49.29\end{array}$ & $\begin{array}{l}2.28 \\
2.17\end{array}$ & $\begin{array}{l}18.87 \\
18.01\end{array}$ & 1698 & $311 \mathrm{~nm}$ & 4.64 \\
\hline $4 e$ & $\begin{array}{c}136-138 \\
96.0 \\
\end{array}$ & $\begin{array}{c}\mathrm{C}_{27} \mathrm{H}_{15} \mathrm{Br}_{2} \mathrm{~N}_{9} \mathrm{O}_{8} \\
753.27 \\
\end{array}$ & $\begin{array}{l}43.05 \\
43.00 \\
\end{array}$ & $\begin{array}{l}2.01 \\
2.00\end{array}$ & $\begin{array}{l}16.74 \\
15.66\end{array}$ & 1700 & $309 \mathrm{~nm}$ & 4.65 \\
\hline $4 f$ & $\begin{array}{c}126-128 \\
93.8\end{array}$ & $\begin{array}{c}\mathrm{C}_{29} \mathrm{H}_{21} \mathrm{~N}_{9} \mathrm{O}_{10} \\
655.54\end{array}$ & $\begin{array}{l}53.13 \\
52.89 \\
\end{array}$ & $\begin{array}{l}3.23 \\
3.11 \\
\end{array}$ & $\begin{array}{l}19.23 \\
18.52 \\
\end{array}$ & 1689 & $307 \mathrm{~nm}$ & 4.49 \\
\hline
\end{tabular}

* ref. (10): $166^{\circ} \mathrm{C}$ 


\section{Acknowledgment}

This research was supported by grant No. CEZ: J 14/98: N7 0000008 of Grant agency of the Czech Republic and by grant No. MSM 153100013 of MSMT ČR.

\section{References}

(1) A. Messmer, A.Gelleri, Angew. Chem. 79. 272 (1967)

(2) J. D. Bower, G. R. Ramage, J. Chem. Soc. 4506, (1957)

(3) R. Pútter:"Methoden zur Herstellung und Unwandlung von Formazanem in Houben-Weyl Methoden der Organische Chemie X/3, p. 631-694"; G. Thieme Verlag Stuttgard (1965)

(4) S. N. Baranov, N. E. Plevachuk - Tarnavskaya, Ukr. Khim. Zh. 29.82 (1963); C. A. $\underline{59}$, 6406e (1963)

(5) Y. Kurasawa, M. Muramatsu, K. Yamazaki. S. Tajima, Y. Okamoto, A. Takada, J. Heter. Chem. 23, 1379 (1986)

(6) Y. Kurasawa, T. Hosaka, K. Ikeda. Y. Matsumoto, A. Ishikura. A. Takada. H. S. Kim. Y. Okamoto, J. Heter. Chem. 31. 527, 1661 (1994)

(7) Y. Kurasawa, T. Hosaka, A. Takada, H. S. Kim, Y. Okamoto, J. Heter. Chem. 32, 445 (1995)

(8) Y. Kurasawa, A. Takano, K. Kato, A. Takada, H. S. Kim, Y. Okamoto, J. Heter. Chem. $33,421(1996)$

(9) I.Wiedermannová, J. Magdonová, J. Slouka, Acta UPO, Fac. Rer. Nat., Chemica $\underline{38,83}$ (1999)

(10) E. Bamberger, J. Müller, J. Pract. Chem. $\underline{64}$, 199 (1901)

Received on July 17, 2000 